\title{
A Three-Dimensional Variational Data Analysis Method with Recursive Filter for Doppler Radars
}

\author{
JIDONG GAO \\ Center for Analysis and Prediction of Storms, University of Oklahoma, Norman, Oklahoma \\ Ming XUE \\ Center for Analysis and Prediction of Storms, and School of Meteorology, University of Oklahoma, Norman, Oklahoma \\ KEITH BREWSTER \\ Center for Analysis and Prediction of Storms, University of Oklahoma, Norman, Oklahoma \\ KeLVin K. Droegemeier \\ Center for Analysis and Prediction of Storms, and School of Meteorology, University of Oklahoma, Norman, Oklahoma
}

(Manuscript received 11 December 2002, in final form 30 July 2003)

\section{ABSTRACT}

\begin{abstract}
In this paper, a new method of dual-Doppler radar wind analysis based on a three-dimensional variational data assimilation (3DVAR) approach is proposed. In it, a cost function, including background term and radial observation term, is minimized through a limited memory, quasi-Newton conjugate-gradient algorithm with the mass continuity equation imposed as a weak constraint. In the method, the background error covariance matrix, though simple in this case, is modeled by a recursive filter. Furthermore, the square root of this matrix is used to precondition the minimization problem.

The current method is applied to Doppler radar observation of a supercell storm, and the analysis results are compared to a conceptual model and previous research. It is shown that the horizontal circulations, both within and around the storms, as well as the strong updraft and the associated downdraft, are well analyzed. Because no explicit integration of the anelastic mass continuity equation is involved, error accumulation associated with such integration is avoided. As a result, the method is less sensitive to the vertical boundary uncertainties.
\end{abstract}

\section{Introduction}

Dual-Doppler wind analysis methods have greatly aided our understanding of weather phenomena ranging from mesoscale convective complexes to clear air boundary layers (e.g., Browning and Wexler 1968; Brandes 1977; Ray et al. 1981; Reinking et al. 1981; Carbone 1983; Kessinger et al. 1987; Parsons and Kropfli 1990; Atkins et al. 1995; Dowell and Bluestein 1997; Sun and Crook 1997, 1998). However, most of these methods still suffer from notable deficiencies, including the setting of often-arbitrary vertical velocity boundary conditions, spatial interpolation and discretization errors, uncertainties in radial wind estimates (due to, e.g., sidelobes and ground clutter), and the nonsimultaneous nature of the measurements. These problems have been

Corresponding author address: Dr. Jidong Gao, Center for Analysis and Prediction of Storms, 100 East Boyd, Suite 1110, Norman, OK 73019.

E-mail: jdgao@ou.edu discussed in Miller and Strauch (1974), Ray et al. (1975), Doviak et al. (1976), Gal-Chen (1982), Testud and Chong (1983), Chong et al. (1983a,b), Ziegler et al. (1983), and Shapiro and Mewes (1999).

The most pronounced difficulties among the above concern the vertical velocity boundary condition and interpolation procedure. The natural boundaries for the problem are the irregular boundaries of the data region itself. If the region of dual-Doppler data coverage extends all the way to the ground, then the impermeability condition can safely be applied. Unfortunately, the lower data boundary often lies hundreds to thousands of meters above ground level, where it is often inappropriate to apply the impermeability condition directly.

When the analysis is performed in a special cylindrical polar coordinate system, termed as the coplane coordinate system by Lhermitte and Miller (1970), it usually includes two steps: interpolation from a radar spherical coordinate system to the coplane coordinate system, and interpolation from a coplane coordinate sys- 
tem to a Cartesian coordinate system after the analysis is completed. Because of the irregular spatial distribution of the radar observations, large intervals between elevation angles [as large as $5^{\circ}-6^{\circ}$ for Weather Surveillance Radar-1988 Doppler (WSR-88D) data], and the presence of bad data values, significant error can be introduced in the spatial interpolation processes.

Gao et al. (1999) proposed a variational method that performs analysis in a Cartesian coordinate system and permits flexible use of radar data in combination with other information [e.g., soundings, or a vertical profile obtained with the velocity-azimuth display (VAD) method]. Furthermore, it allows for the use of mass continuity and smoothness constraints by incorporating them into a cost function. In particular, by applying the anelastic mass conservation equation as a weak constraint, the severe error accumulation in the vertical velocity can be reduced because the explicit integration of the anelastic continuity equation is avoided. The method performs well in both idealized Observing System Simulation Experiments (OSSEs) and real data cases. However, there exist some difficulties in specifying the optimal weighting for each constraint. In the previous study, weightings were selected based on past experience and repeated tuning experiments. Finding the optimal weighting for each constraint may require many numerical experiments.

Proposed herein is a new technique, based on a standard three-dimensional variational data assimilation (3DVAR) approach, whereby a background error covariance matrix, though simple, is modeled by a recursive filter, and the square root of the matrix is used for preconditioning. Using the recursive filter is a simple and efficient way to spread the effect of each radar observation to the analyzed grid points (Wu et al. 2002). Recent developments with spatially recursive filters (Purser et al. 2003a) enable the construction of a variational analysis in physical space, which allows more degrees of freedom in defining the error statistics adaptively. The eventual goal is to have an analysis system with inhomogeneous and generally anisotropic threedimensional background error covariance. Compared to the smoothness constraint used in Gao et al. (1999), the recursive filter is more effective in achieving the desired spreading of observations to nearby grid points, and theory (Purser et al. 2003a) provides guidance for specifying filter coefficients.

The aim of the preconditioning procedure is to decrease the number of iterations in the minimization process for obtaining the optimal solution of the analysis. The weight assigned to each constraint is specified according to its assumed error magnitude, so that each weight has its physical meaning rather than being chosen somewhat arbitrarily based on experience and iterative experiments.

Because the method combines interpolation and analysis into a single step, as with the earlier variational scheme of Gao et al. (1999), the analysis is performed more naturally and directly in a Cartesian coordinate system; only an interpolation from the regular Cartesian grid to the irregular radar observation points is needed. Since this interpolation process is usually well defined, the error should be smaller than when interpolation is performed from an irregular radar coordinate system to a regular Cartesian coordinate system. Further, this reverse interpolation procedure accurately preserves the radial nature of radar observations.

This paper is organized as follows. In section 2 , the variational method is introduced. In section 3 , the basic capabilities of the recursive filter are tested by placing a single observation in the center of model domain. The developed method is then applied to the analysis of a dual-Doppler dataset from an observed tornadic supercell storm. Finally, a summary and concluding remarks are given in section 4 .

\section{Description of variational methodology}

\section{a. Formulation of cost function}

The standard formulation of variational methods was derived from first principles by Lorenc using Bayesian probabilities and assuming Gaussian error distributions (Lorenc 1986). The concept of a variational method is to determine the analysis by direct minimization of a cost function. The gradients of cost function must be derived to determine the search direction in the minimization. The cost function may be written as

$$
\begin{aligned}
J(x)= & J_{B}+J_{O}+J_{C} \\
= & \frac{1}{2}\left(x-x_{b}\right)^{\mathrm{T}} \mathbf{B}^{-1}\left(x-x_{b}\right) \\
& +\frac{1}{2}\left[H(x)-y_{o}\right]^{\mathrm{T}} \mathbf{R}^{-1}\left[H(x)-y_{o}\right]+J_{C} .
\end{aligned}
$$

In its simplest form, the cost function, $J$, may be written as the sum of two quadratic terms: the first, $J_{B}$, measures the departure of the analysis or control variable, $x$, from the background, $x_{b}$, and is weighted by the inverse of the background error covariance matrix B; the second term, $J_{o}$, measures the departure of the analysis $x$, at the observation locations by means of interpolation operator $H$, from the observations $y_{O}$, and is weighted by the inverse of the observational error covariance matrix R. To impose additional physical constraints among the analysis variables, a dynamic or smoothness constraint term, $J_{C}$, can be added to the cost function. This term is also called the penalty term.

The goal of the analysis is to find state, $x_{a}$, or the solution of $x$, for which $J$ is minimized. At the minimum, the derivative of $J$ vanishes, and the so-called optimal estimate, $x_{a}$, satisfies

$$
\begin{aligned}
\boldsymbol{\nabla} J(x)= & \mathbf{B}^{-1}\left(x-x_{b}\right)+\mathbf{H}^{\mathrm{T}} \mathbf{R}^{-1}\left[H(x)-y_{o}\right] \\
& +\boldsymbol{\nabla} J_{C}(x)=0
\end{aligned}
$$


where $\mathbf{H}$ is a suitable linear approximation operator of $H$ in the vicinity of $x_{b}$.

The second-order derivative or the Hessian of $J(x)$ can be derived as

$$
\nabla^{2} J(x)=\mathbf{B}^{-1}+\mathbf{H}^{\mathrm{T}} \mathbf{R}^{-1} \mathbf{H}+\nabla^{2} J_{C}(x) .
$$

If $\nabla^{2} J(x)$ is positive definite, then there exists a unique $x_{a}$ that minimizes the cost function $J(x)$.

\section{b. Preconditioned control variable}

Following Courtier et al. (1994) and Courtier (1997), suppose the optimum analysis $x_{a}$ can be obtained by adding optimal analysis increment $\delta x_{a}$ to the background $x_{b}$ :

$$
x_{a}=x_{b}+\delta x_{a} ;
$$

then the cost function can be rewritten as

$$
\begin{aligned}
J_{\text {inc }}(\delta x)= & \frac{1}{2}(\delta x)^{\mathrm{T}} \mathbf{B}^{-1}(\delta x) \\
& +\frac{1}{2}(H \delta x-d)^{\mathrm{T}} \mathbf{R}^{-1}(H \delta x-d)+J_{C}(\delta x),
\end{aligned}
$$

where $d \equiv y_{o}-H x_{b}$ is the innovation vector and subscript "inc" for $J$ denotes "incremental." The background error covariance matrix B can be regarded as an operator and is constructed using its square root: $\mathbf{B}=$

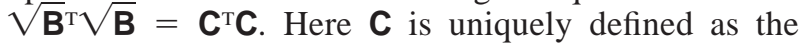
symmetric matrix that has the same eigenvectors as $\mathbf{B}$ and eigenvalues of the square root of those of $\mathbf{B}$.

An effective way of preconditioning the minimization procedure is to define an alternative control variable $q$, according to

$$
q=\mathbf{C}^{-1} \delta x \text { or } \delta x=\mathbf{C} q .
$$

Then Eq. (5) becomes

$$
J_{\text {inc }}(q)=\frac{1}{2} q^{\mathrm{T}} q+\frac{1}{2}(\mathbf{H C} q-d)^{\mathrm{T}} \mathbf{R}^{-1}(\mathbf{H C} q-d)+J_{C}(q) .
$$

The gradient and Hessian of $J_{\text {inc }}$ can also be derived, with the former obtained by differentiating Eq. (7) with respect to $q$ :

$$
\begin{aligned}
\boldsymbol{\nabla} J_{\text {inc }}(q)= & \left(\mathbf{I}+\mathbf{C}^{\mathrm{T}} \mathbf{H}^{\mathrm{T}} \mathbf{R}^{-1} \mathbf{H C}\right) q \\
& -\mathbf{C}^{\mathrm{T}} \mathbf{H}^{\mathrm{T}} \mathbf{R}^{-1} \mathbf{d}+\boldsymbol{\nabla} J_{C}(q) .
\end{aligned}
$$

The Hessian follows as

$$
\nabla^{2} J_{\text {inc }}=\mathbf{I}+\mathbf{C}^{\mathrm{T}} \mathbf{H}^{\mathrm{T}} \mathbf{R}^{-1} \mathbf{H C}+\nabla^{2} J_{C}(q),
$$

where $\mathbf{I}$ is the identity matrix. Comparing Eqs. (9) and (3), it is evident that the preconditioning resulting from (9) prevents the smallest eigenvalue from becoming less than unity, while there is no guarantee of such with the Hessian given in Eq. (3). Typical minimization algorithms converge faster when the condition number, defined as the quotient between the largest and smallest eigenvalues of the Hessian matrix, is smaller. Thus, this new Hessian matrix is much better conditioned than the Hessian of the original problem (3).

The matrix $\mathbf{C}$ defined in (6) can be broken down as

$$
\mathbf{C}=\mathbf{D F},
$$

where $\mathbf{D}$ is a diagonal matrix of the standard deviation of the background error $\mathbf{B}$ and $\mathbf{F}$ is the square root of a matrix whose diagonal elements are equal to one, and off-diagonal elements are the background error correlation coefficients. In practical data assimilation for numerical weather prediction (NWP), full matrix $\mathbf{F}$ is too large to compute explicitly or store into computer memory. Assumptions and approximations are usually made. It can be shown that the effect of applying operator $\mathbf{F}$ on control variable $q$ in Eq. (7) can be achieved through the use of equivalent spatial filters (Huang 2000). Here we follow the work of Purser and McQuigg (1982), Lorenc (1992), and Hayden and Purser (1995), who model the effect of $\mathbf{F}$ using a recursive filter that is defined by

$$
\begin{array}{ll}
Y_{i}=\alpha Y_{i-1}+(1-\alpha) X_{i} & \text { for } i=1, \ldots, n \\
Z_{i}=\alpha Z_{i+1}+(1-\alpha) Y_{i} & \text { for } i=n, \ldots, 1,
\end{array}
$$

where $X_{i}$ is the initial value at grid point $i, Y_{i}$ is the value after filtering for $i=1$ to $n, Z_{i}$ is the initial value after one pass of the filter in each direction, and $\alpha$ is the filter coefficient given by the following formulation (Lorenc 1992):

$\alpha=1+E-\sqrt{E(E+2)}, \quad E=2 N \Delta x^{2} /\left(4 L^{2}\right)$,

where $L$ is the horizontal correlation scale, $\Delta x$ is the grid spacing, and $N$ is the number of filter passes to be applied. This is a first-order recursive filter, applied in both directions to ensure zero phase change. Multipass filters ( $N$ greater than unity) are built up by repeated application of (11). Two- and three-dimensional filters can be constructed by applying this filter successfully in each direction. It can be shown (Purser et al. 2003a) that such multidimensional filters, when applied with several passes, can accurately model isotropic Gaussian error correlations. The filter is also flexible enough to allow for anisotropic error correlations (Purser et al. 2003b).

For simplification, we assume that the observation error covariance matrix $\mathbf{R}$ is diagonal with constant diagonal elements given by the estimated observation error, which is $1 \mathrm{~m} \mathrm{~s}^{-1}$ for typical radar observations (Miller and Sun 2003; Xu and Gong 2003).

\section{c. Variational dual-Doppler radar data analysis}

In our variational dual-Doppler wind analysis, the first term, $J_{B}$, in the cost function measures how close the variational analysis $x=(u, v, w)$ fits the background fields $x_{b}=\left(u_{b}, v_{b}, w_{b}\right)$. The background may be provided by a previous model forecast, a nearby sounding, 
or a wind profile from another Doppler radar analysis program, such as the VAD method (Browning and Wexler 1968). The second term, $J_{O}$, measures the difference between the analyzed radial velocity, $V_{r}$, which can be approximated (for distance between radar site and data point $r$ less than $100 \mathrm{~km}$ ) as

$$
V_{r}=\frac{\left(X-X_{0}\right) u+\left(Y-Y_{0}\right) v+\left(Z-Z_{0}\right) w}{r},
$$

and the observed radial velocity $y_{0}=V_{\text {rob }}$. The forward operator, $H(x)$ in this case, is represented by Eq. (13) and a linear interpolation operator that maps $V_{r}$ from the grid (Cartesian coordinates) to observation points (spherical coordinates); $u, v$, and $w$ are wind components in Cartesian coordinates $(X, Y, Z)$; and $\left(X_{0}, Y_{0}, Z_{0}\right)$ is the radar location. It should be noted that the cost function could include other observations that directly or indirectly provide information about $u, v$, and $w$, such as single-level data from surface observations or data from wind profilers, aircraft, or even satellites if these data could be beneficial to the analysis.

The third term, $J_{C}$, can be expressed as

$$
J_{C}=\frac{1}{2} \lambda_{c} D^{2}
$$

which imposes a weak anelastic mass continuity constraint on the analyzed wind field, where

$$
D=\partial \bar{\rho} u / \partial x+\partial \bar{\rho} v / \partial y+\partial \bar{\rho} w / \partial z,
$$

and $\bar{\rho}$ is the mean air density at a given horizontal level. The weighting coefficient, $\lambda_{C}$, controls the relative importance of this penalty term in the cost function. To specify $\lambda_{C}$, the radar-observed radial velocities are projected in the $x, y$, and $z$ directions to obtain an estimate of the $u, v$, and $w$ fields. These partially measured wind fields then are applied to assess the divergence, and $\lambda_{C}$ is given by (Liou 1999)

$\lambda_{C}=\left[\frac{1}{\Omega} \int_{\Omega}\left(\frac{\partial \bar{\rho} u}{\partial x}+\frac{\partial \bar{\rho} v}{\partial y}+\frac{\partial \bar{\rho} w}{\partial z}\right)^{2} d x d y d z\right]^{-1}$,

where $\Omega$ is the analysis domain.

To solve the above variational problem by direct minimization, we need to derive, by using Eq. (8), the gradients of the cost function with respect to the control variables, which contain the increments of three wind components transformed to $q$ according to Eq. (6). After the gradients of the cost function are obtained, the problem can be solved in the following steps.

1) Choose the first guess of control variables $q$ (in our experiments, all first guess increments are zero).

2) Calculate the cost function according to Eq. (7) and the gradient according to Eq. (8).

3) Use a conjugate-gradient method (Navon and Legler 1987) to obtain updated values of the control variables,

$$
q^{(n)}=q^{(n-1)}+\beta f\left(\partial J_{\mathrm{inc}} / \partial q\right)
$$

where $n$ is the number of iterations, $\beta$ is an optimal step size obtained by the so-called line search process in the optimal control theory (Gill et al. 1981), and $f(\partial J / \partial q)$ is the optimal descent direction obtained by combining the gradients from several previous iterations.

4) Check if the optimal solution has been found by computing the norm of the gradients or the value of $J$ itself to see if either is less than a prescribed tolerance (we choose $J / J_{O}=10^{-3}$, and $J_{O}$ is the cost function for the first iteration). If the convergence criterion is not satisfied, repeat steps 2 and 3 using updated values of $q$ as the new guess. The iteration process is continued until a prescribed convergence criterion is met or a maximum iteration number is reached (300 here).

5) Finally, recover the analysis variables, $u, v$, and $w$, from $q$ using Eq. (6).

\section{d. The effect of terminal velocity}

Before applying the above variational method, the fall speed of precipitation has to be taken into account when radial velocity data are used. For radar scans at nonzero elevation angles, the fall speed contributes to the Doppler estimate of radial velocity. Thus, the observations of radial velocity are adjusted to remove this contribution using

$$
V_{r}=V_{r}^{a}+w_{t} \sin \theta,
$$

where $V_{r}^{a}$ is the radial velocity actually observed by the radar, $V_{r}$ is the true radial velocity of the air, $w_{t}$ is the terminal velocity of precipitation, and $\theta$ is the elevation angle $\left(0^{\circ}\right.$ is horizontal). An empirical relationship is used to relate the reflectivity, $R$, and raindrop terminal fall velocity (Foote and du Toit 1969; Atlas et al. 1973):

$$
w_{t}=2.65\left(\frac{\rho_{0}}{\rho}\right) R^{0.114},
$$

where $\rho$ is the air density and $\rho_{0}$ is its surface value. Note that, in this formulation, $w_{t}$ is positive downward.

\section{Experiment results}

To demonstrate the effectiveness of the described variational method for real data, we apply it to the 17 May 1981 Arcadia, Oklahoma, supercell storm (Dowell and Bluestein 1997). Twelve coordinated dual-Doppler scans were obtained from the Norman and Cimarron, Oklahoma, S-band Doppler radars over the 1-h period spanning the pretornadic phase of the storm. The analysis is performed in a Cartesian coordinate system with $83 \times 83 \times 37$ grid points. The grid spacing in the horizontal is $\Delta x=\Delta y=1000 \mathrm{~m}$ and in the vertical is $\Delta z=500 \mathrm{~m}$. The standard deviation of errors for the radar radial velocity is set to $1 \mathrm{~m} \mathrm{~s}^{-1}$, and the standard deviation of background errors (only a single sounding 


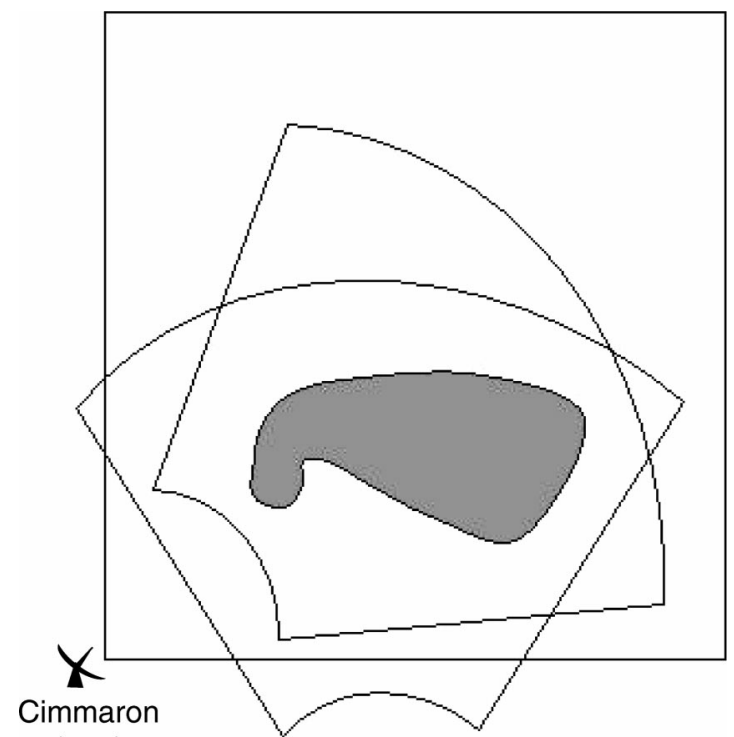

$(0,0)$

\section{$\mathbf{X}_{(40,-20)}^{\text {Norman }}$}

FIG. 1. Locations of the Cimarron $(0,0)$ and Norman $(40.0,-20.0)$, OK, radars that observed the 17 May 1981 Arcadia, OK, storm (shading) and the analysis domain (box).

is used in this analysis) is taken to be $10 \mathrm{~m} \mathrm{~s}^{-1}$. The analysis domain and the relative positions of two radars are shown in Fig. 1. To clearly show the behavior of the analysis method, we perform four experiments using a single observation within the analysis domain before applying the method to the observed supercell storm with several full volume scans.

\section{a. Single-observation experiment}

In our first experiment, we place only one radial velocity observation with $V_{r}=26.60 \mathrm{~m} \mathrm{~s}^{-1}$ relative to Cimmaron radar within the analysis domain at a selected gridpoint location $(42.70,29.90,9.60)$ and exclude the mass continuity constraint from the minimization process $\left(J_{C}=0.0\right)$. Figure 2 illustrates the spread of the information from this single observation over the one pass with $N=1$ and a correlation scale $L=5 \Delta x$ defined by the first-order recursive filter in (11) and (12). Note that the analysis increments of both horizontal velocity $u$ and vertical velocity $w$ exhibit diamond shapes, while the desired shape in the absence of any equation constraint is circular.

Since the single radial velocity observation is placed at $z=9 \mathrm{~km}$, it also contributes significantly to the vertical velocity (Fig. 2b) with a maximum of $5 \mathrm{~m} \mathrm{~s}^{-1}$ in the grid cell nearest the single observation. When the recursive filter is applied two times $(N=2)$, the result is closer to being isotropic but still not perfectly so (Fig. 3 ). The appearance of isotropy is not adequately (a)

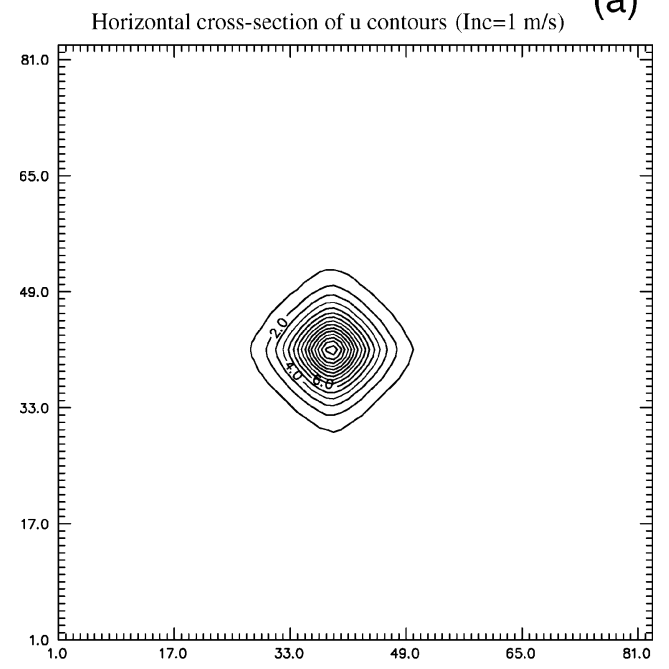

(b)

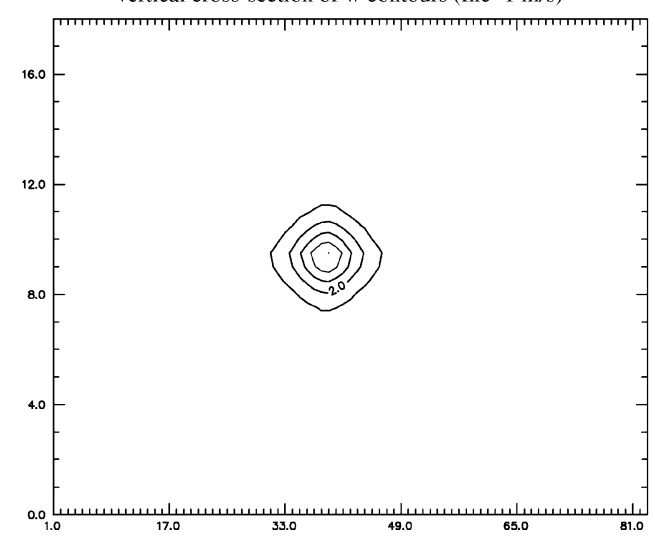

FIG. 2. Wind contours demonstrating the application of the recursive filter to a single radial velocity $\left(26 \mathrm{~m} \mathrm{~s}^{-1}\right)$ observation relative to the Cimmaron radar with iteration $N=1$ and correlation scale $L_{h}$ $=5 \Delta x$ and $L_{z}=5 \Delta z$ : (a) the response function of $u$ in a horizontal cross section; (b) $w$ in vertical cross section. Distance scale in $\mathrm{km}$.

achieved until $N=4$ (Fig. 4). This shows that the firstorder recursive filter can only provide an excellent approximation to the isotropic Gaussian structure with four or more passes. Therefore, we use four passes in the following experiments.

It is also necessary to properly choose a correlation scale length in our scheme. If it is too large, the analyzed field will be too smooth and the analyzed mesocyclone and mesoanticyclone will be too weak. If it is too small, the analysis wind fields will lack coherent structure because the gap between the neighboring tilts cannot be filled. Here, using $L=5 \Delta x$ for the horizontal scale and $L=5 \Delta z$ for the vertical, given both radars' scanning sequences and parameter settings, has been found to produce credible results. Additionally, this feature is very well preserved as the number of passes increases, as indicated by the analyses shown in Figs. 2-4. 
Horizontal cross-section of $\mathrm{u}$ contours ( $\mathrm{Inc}=1 \mathrm{~m} / \mathrm{s}$ )

(a)

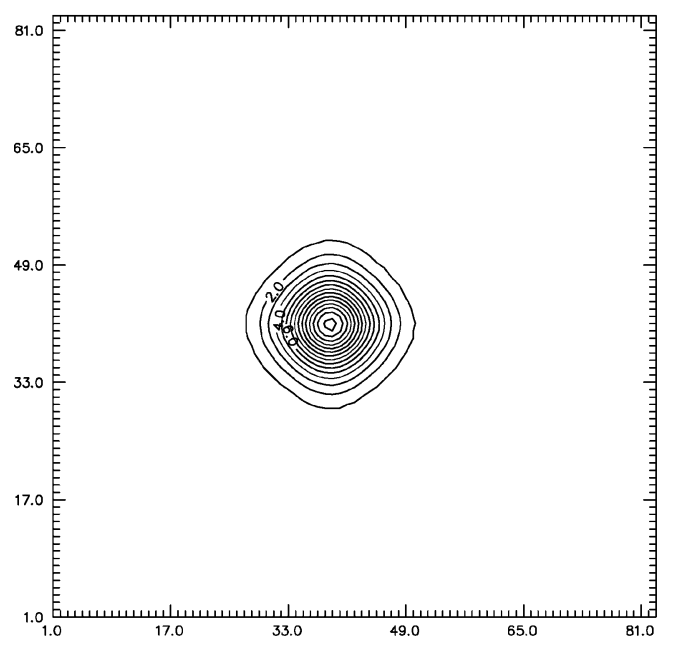

(b)

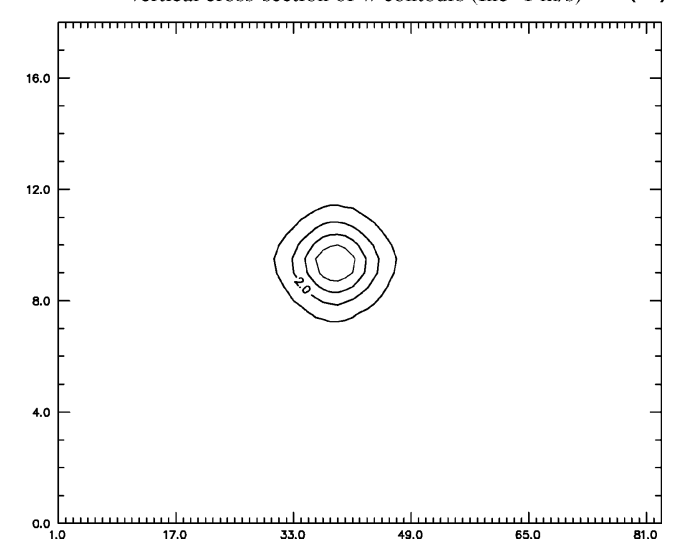

FIG. 3. The same as Fig. 2, but for $N=2$.

When the anelastic mass continuity equation is added to the cost function as a weak constraint, the response covariance function of this single observation differs from previous cases in both the horizontal wind and vertical velocity (Fig. 5), both of which are no longer isotropic. This result is more realistic, however, because the mass continuity equation builds up an appropriate coupling among the three wind components. For example, in the analyzed $w$ field, instead of a single region of positive $w$ in response to one positive radial velocity measurement, there is now one positive region as well as one negative region of $w$. This structure represents the response of the flow to mass conservation. If more data are used, the relationship among analysis variables should be more coherent than without using it. Furthermore, additional equation constraints that couple the wind with other fields can be added, and it is possible
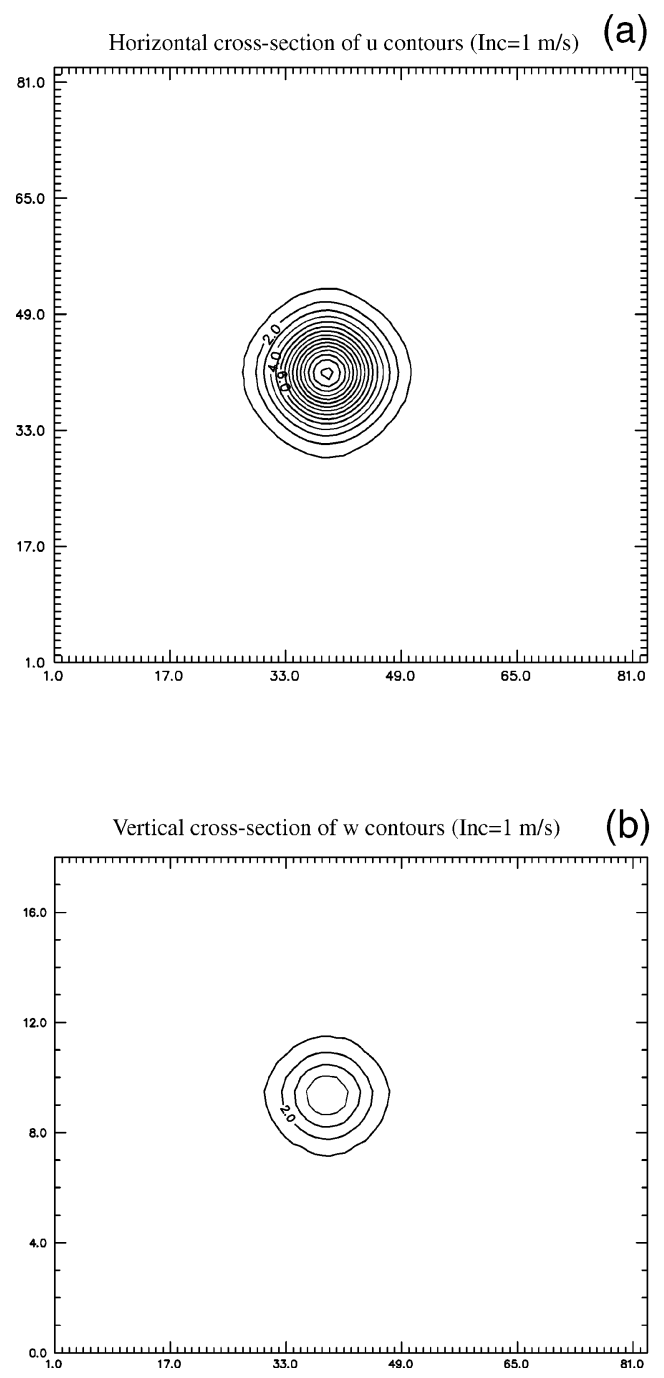

FIG. 4. The same as Fig. 2, but for $N=4$.

to incorporate flow-dependent anisotropic spatial correlations into the analysis through a recursive filter. A better and more complete analysis could be expected with these improvements, and work is under way along these lines.

\section{b. Application to a real supercell storm case}

In this section, we apply the method described above to the complete dataset for the 17 May 1981 Arcadia supercell storm. Using the dual-Doppler analysis program contained in the National Center for Atmospheric Research (NCAR) software package CEDRIC (Mohr 1988), Dowell and Bluestein (1997) performed a detailed dual-Doppler analysis of this storm that will serve as our benchmark. Using the same dataset, we also performed a dual-Doppler analysis using a variational package developed by ourselves (Gao et al. 1999). Due to the lack of more radar data, neither this nor the Dowell 
(a)

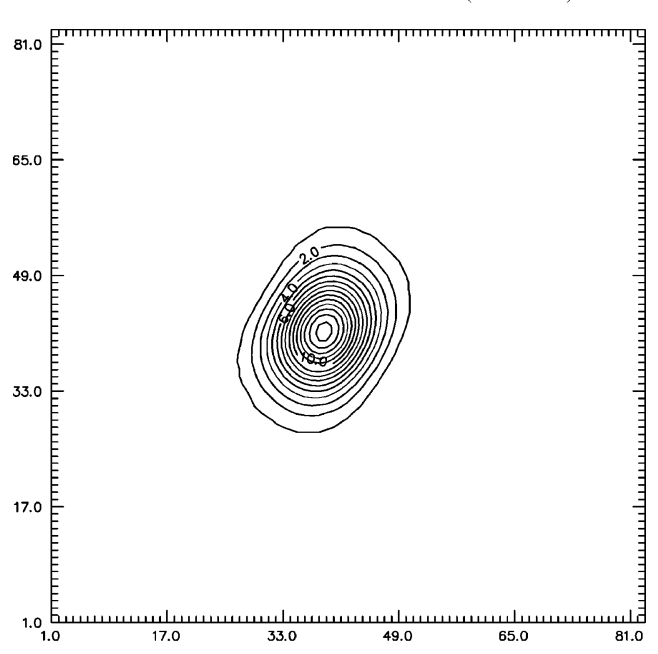

(b)

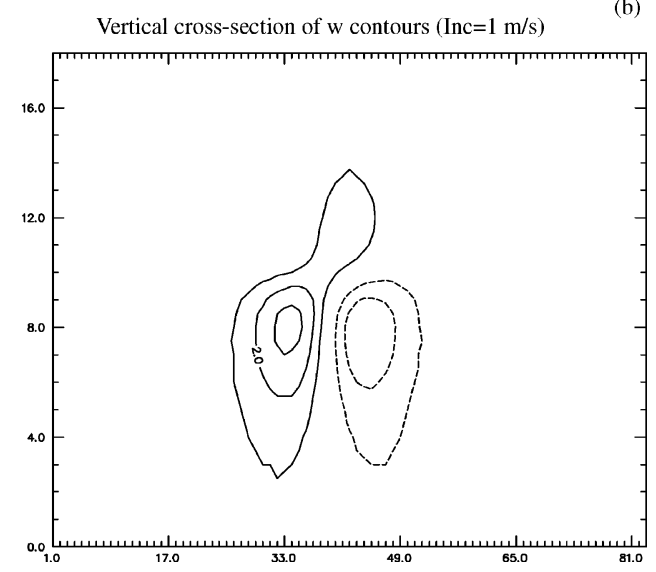

FIG. 5. The same as Fig. 2, but for $N=4$ and with mass conservation equation as a weak constraint.

and Bluestein analysis can be validated against a more accurate analysis. Therefore, the present discussion remains qualitative, and the analysis results are compared with a conceptual model and previous research.

The results of our analysis for 1634, 1638, and 1643 central standard time (CST) on 17 May are shown in Figs. 6, 7, and 8, respectively. During this period, the low-level precipitation echo evolved from a north-south elongated pattern (Fig. 6a) into one with a pronounced hook shape (Fig. 8a) as the low-level rotation intensified. The presence of such a hook-echo pattern in the reflectivity field signifies the transition of the supercell storm to the tornadic stage (Brandes 1981). The advancement of the gust front from west to east, pushed by cold outflow behind, is clearly seen from these figures. Ahead (east) of the gust front, the analyzed lowlevel flow shows strong southerly to southeasterly winds, and the flow turns to easterly as it reaches northeast, then the north side of the center of rotation (me-
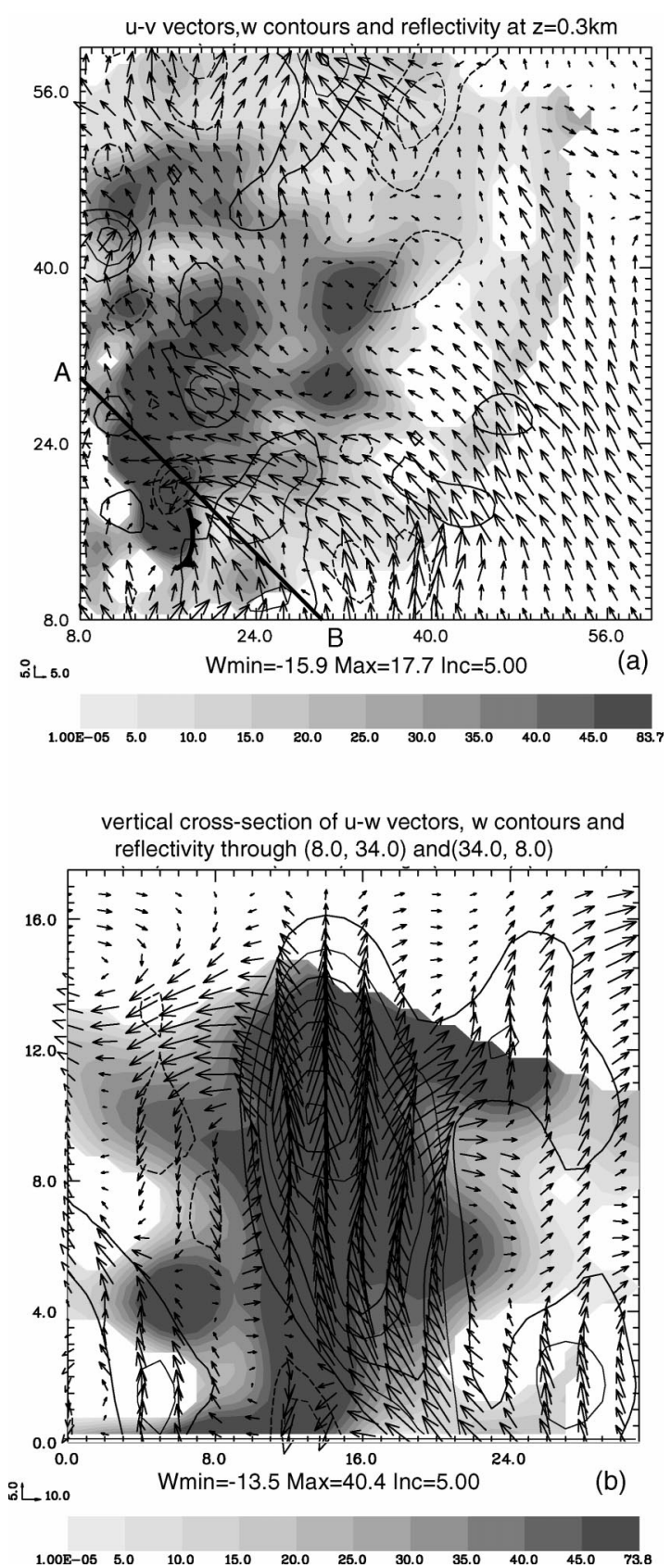

FIG. 6. Wind vectors and vertical velocity (contours) analyzed from data sampled by Norman and Cimarron radars using the variational method for Arcadia, OK, at 1634 CST 17 May 1981 tornadic storm. Also shown as shaded contours of the reflectivity field. (a) Horizontal cross section at $z=0.3 \mathrm{~km}$. (b) Vertical cross section through the A-B line in (a). The rear-flank gust front at this level is indicated by the cold front symbol in (a). Radar observations are only available where there is reflectivity shading, and winds shown are relative to the storm, which was moving from $240^{\circ}$ at $11.7 \mathrm{~m} \mathrm{~s}^{-1}$.

socyclone), which roughly coincides with the kink of the hook echo. Some of this flow eventually turns southward on the northwest and west sides and feeds into the outflow behind the gust front. 

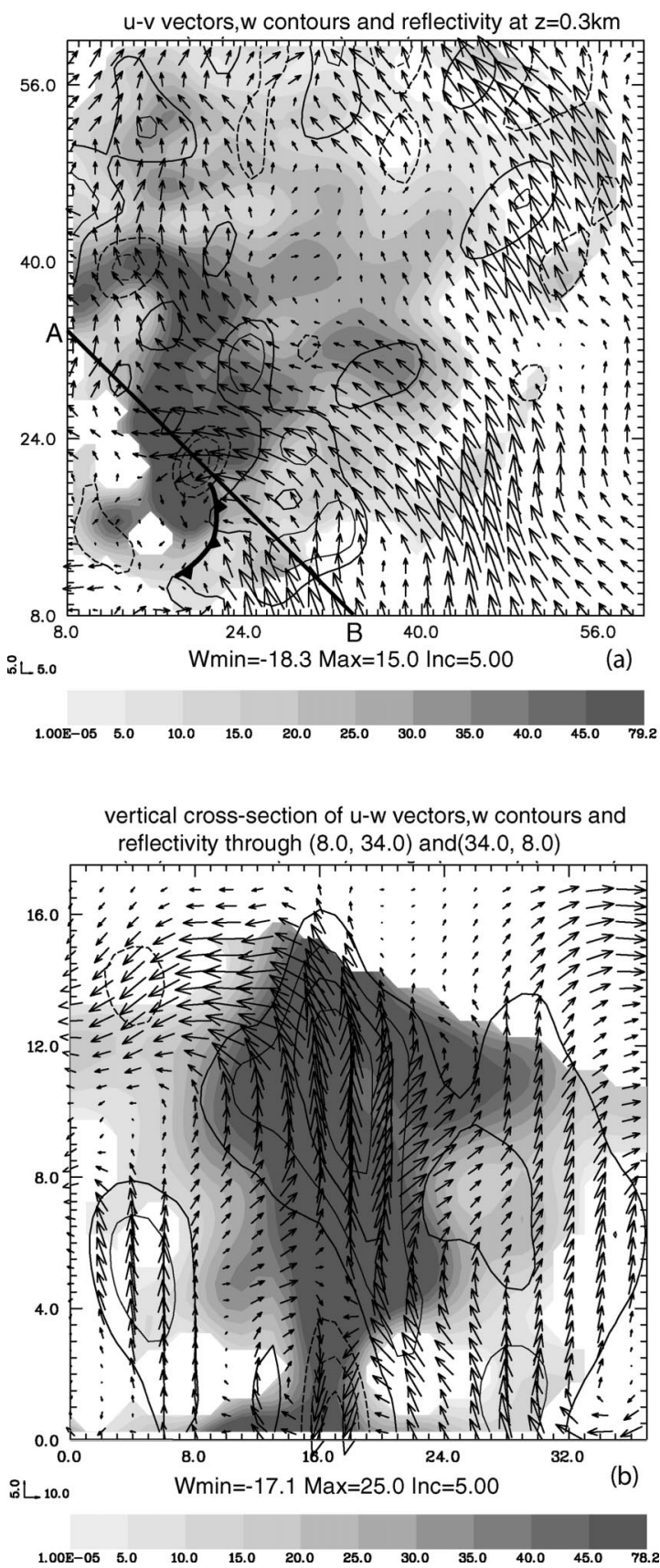

FIG. 7. As in Fig. 6, but for 1638 CST.

Strong convergence in the near-surface flow is found within $10 \mathrm{~km}$ east of the gust front, where positive vertical motion at $300 \mathrm{~m}$ above ground is analyzed to reach more than $10 \mathrm{~m} \mathrm{~s}^{-1}$ at the first two times (1634 and 1638 CST) and increases to more than $20 \mathrm{~m} \mathrm{~s}^{-1}$ by 1643 CST, at which time two maxima are found within $5 \mathrm{~km}$ of the gust front (Fig. 8a). Also found in the vertical velocity field is a downdraft maximum near the kink of the hook echo and on the west side of the occlusion point of the forward- and rear-flank gust fronts (only
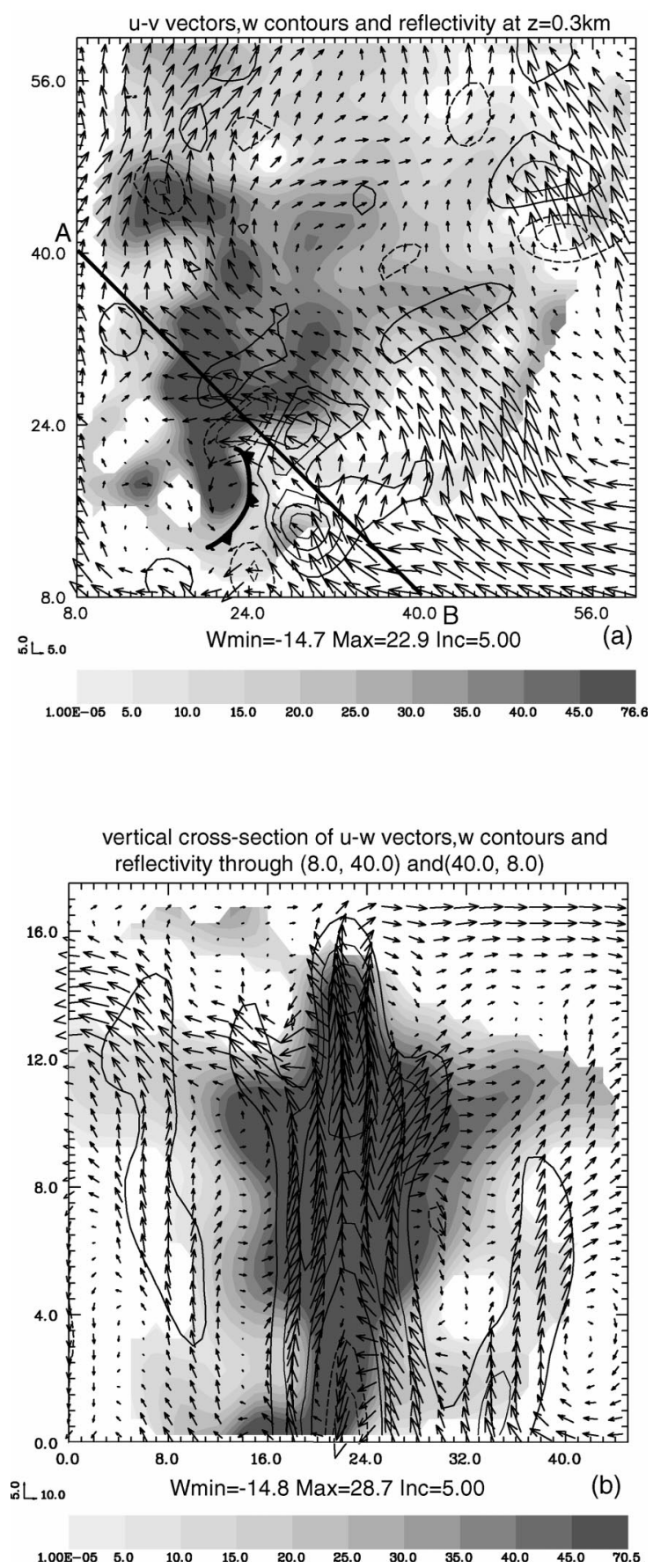

FIG. 8. As in Fig. 6, but for 1643 CST.

the latter is drawn in the figures). This downdraft appears realistic because of the time continuity among all three analyses and is consistent with the rear-flank downdraft commonly found in tornadic supercells.

Overall, the analyzed low-level flow structures shown in these figures are consistent with the reflectivity patterns and are typical of tornadic supercell storms with strong low-level rotation (e.g., Lemon and Doswell 1979). 
In a vertical cross section (Fig. 6b), plotted through line A-B in Fig. 6a, a strong updraft core is retrieved and is roughly collocated with the midlevel mesocyclone (center of strong rotation) and the core of maximum reflectivity. The main updraft is seen to originate ahead of the low-level gust front and reaches a height of $16 \mathrm{~km}$. It gains a maximum intensity of $40.4 \mathrm{~m} \mathrm{~s}^{-1}$ at $10 \mathrm{~km}$ in this cross section and is, as expected, near the area of maximum reflectivity aloft. The main downdraft is located below the updraft core and is collocated with a region of high reflectivity behind the gust front. At a later time (Fig. 7b), it is clearly shown that a very strong downdraft begins to develop beneath the updraft core between $4 \mathrm{~km}$ and the ground. Figure $8 \mathrm{~b}$ shows that the downdraft is encircled by a larger updraft. This structure is realistic and typical and represents a transition of the supercell to the tornadic phase (Houze and Hobbs 1982). At this time, a downdraft forms at the center of an earlier updraft core, a process explained clearly in the review article of Klemp (1987), a figure from which is reproduced in Fig. 9. According to this article, it is believed that a downward pressure gradient force created by the reduction of low-level pressure as a result of intensification of low-level rotation is believed to be the cause of downdraft intensification.

Because this analysis is valid about several minutes before the tornadogenesis, the large horizontal shear caused by the narrow downdraft and surrounding updraft could be the source of development of the subsequent tornadic vortex. These features, including a decrease in updraft intensity (reduced from about 40 to $25 \mathrm{~m} \mathrm{~s}^{-1}$ from Fig. 6b to Fig. 7b), a small-scale downdraft forming underneath the updraft, and a flow at low levels in which cold-outflow and warm-inflow air spiral around the center of circulation, suggest that both horizontal and vertical flows are kinematically consistent. Similar features were also observed and documented quite well by Lemon and Doswell (1979) in their case studies. They also qualitatively agree with those analyzed by Dowell and Bluestein (1997) and Gao et al. (1999) but provide more detailed structures of this tornadic storm.

When radar data are to be used to initialize an NWP model, a complete description of the wind and other meteorological variables is needed for the entire model domain (Droegemeier 1997). Even for diagnostic studies, consistent analysis outside the radar data areas is also desirable. Conventional observations such as from rawinsondes, or background fields from NWP models, can be used to complement radar data. However, the radar data must be blended with the background information.

Lin et al. (1993) proposed a method for obtaining a smooth transition from regions with radar observations to the ambient environment defined by a single sounding. Ellis (1997) also used an OSSE to fill the data voids (hole filling) in simulated radar data. In both cases a variational wind adjustment procedure was then applied
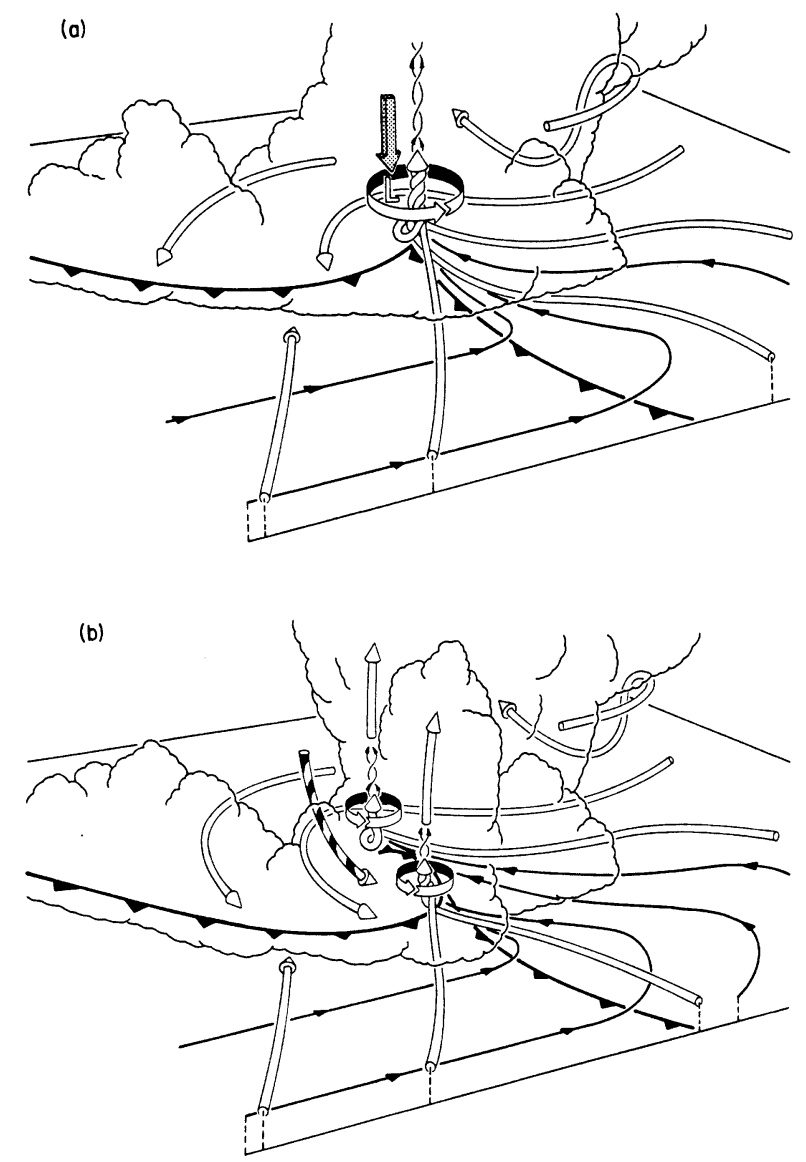

FIG. 9. Three-dimensional perspective of a numerically simulated supercell thunderstorm as it transitions to its tornadic phase. The storm is evolving in westerly environmental wind shear and is viewed from the southeast. The cylindrical arrows depict the flow in and around the storm. The thin lines show the low-level vortex lines, with the arrows along the lines indicating the vector direction of vortex. The heavy barbed line marks the boundary of cold air beneath the storm. (a) The stage at which a strong downdraft starts to form and (b) about 10 min later. The shaded arrow in (a) represents the rotationally induced vertical pressure gradient, and the striped arrow in (b) denotes the rear-flank downdraft. (From Klemp 1987.)

to the hold-filled wind field. The variational wind adjustment improves the statistics of each hole-filled wind field. In Gao et al. (1999), a single sounding was used to specify the background environment. In that study, although no observational constraint was available outside the radar-observed data areas, the analysis took on the values of the background and the transition was smooth between the data area and nondata area, owing to the use of a smoothness constraint.

In the current method, observational information is spread by means of the background error covariance that is modeled by a recursive filter, which in its current form is isotropic and homogeneous. As noted previously, the standard error deviation for radar radial velocity observations is set to $1 \mathrm{~m} \mathrm{~s}^{-1}$ and that for the background sounding is set to $10 \mathrm{~m} \mathrm{~s}^{-1}$. By doing so, we ensure that radar observations dominate in the region 
containing radar observations and that the background plays only a marginal role in that area. However, where no radar observations exist, the analyses would take on the values of background field. The transition between the two is more natural in that variational data assimilation optimally blends observations with the background.

\section{c. The role of the anelastic mass continuity constraint}

Our variational method produces satisfactory results for 3D dual-Doppler wind analysis, including the vertical velocity. To clearly show the role of the weak mass continuity constraint with the real data, we perform an experiment that is identical to the control case (the above) except that the mass continuity constraint is removed. As shown in Fig. 10, the reasonable horizontal wind is retrieved (but not as well as the previous analysis); however, the retrieval of vertical velocity $w$ is very poor, and no coherent structure in $w$ is retrieved below $10 \mathrm{~km}$, though the results above $10 \mathrm{~km}$ are qualitatively good. This result is not surprising, though, because very limited information about $w$ is contained in the radial wind observations at low levels.

With conventional dual-Doppler analysis or retrieval methods, the vertical velocity is obtained by vertically integrating the mass continuity equation; in this sense, the equation is used as a "strong constraint" because the equation is exactly satisfied. The main difficulty lies in specifying vertical boundary conditions on $w$. The requirement of zero vertical velocity at the ground is the most natural physical condition (Miller and Strauch 1974). However, it has been demonstrated that upward integration is unreliable because the bias errors in the divergence field can be amplified exponentially with height (Ray et al. 1980). Thus, a variational wind adjustment was applied to the analyzed winds in their multiple-Doppler radar analysis with the anelastic mass continuity equation used as a strong constraint. Matejka and Bartels (1998) compared eight methods of calculating vertical velocity in a column and found that their two variational methods performed best in many cases, but the methods still have problems with the low boundary condition, which may in part be due to the use of the mass continuity equation as a strong rather than weak constraint.

Vertical integration of the anelastic mass continuity equation is particularly difficult for our current dataset because of the presence of large data voids. The resultant $w$ field from such integration can be very problematic even if an additional variational adjustment is applied. It is therefore advantageous to apply the mass continuity equation as a weak constraint so that explicit integration of the continuity equation is avoided.

To summarize, in conventional dual-Doppler analysis techniques, the horizontal and vertical winds are derived in two separate steps. With our method, the horizontal and vertical wind components are analyzed in a single
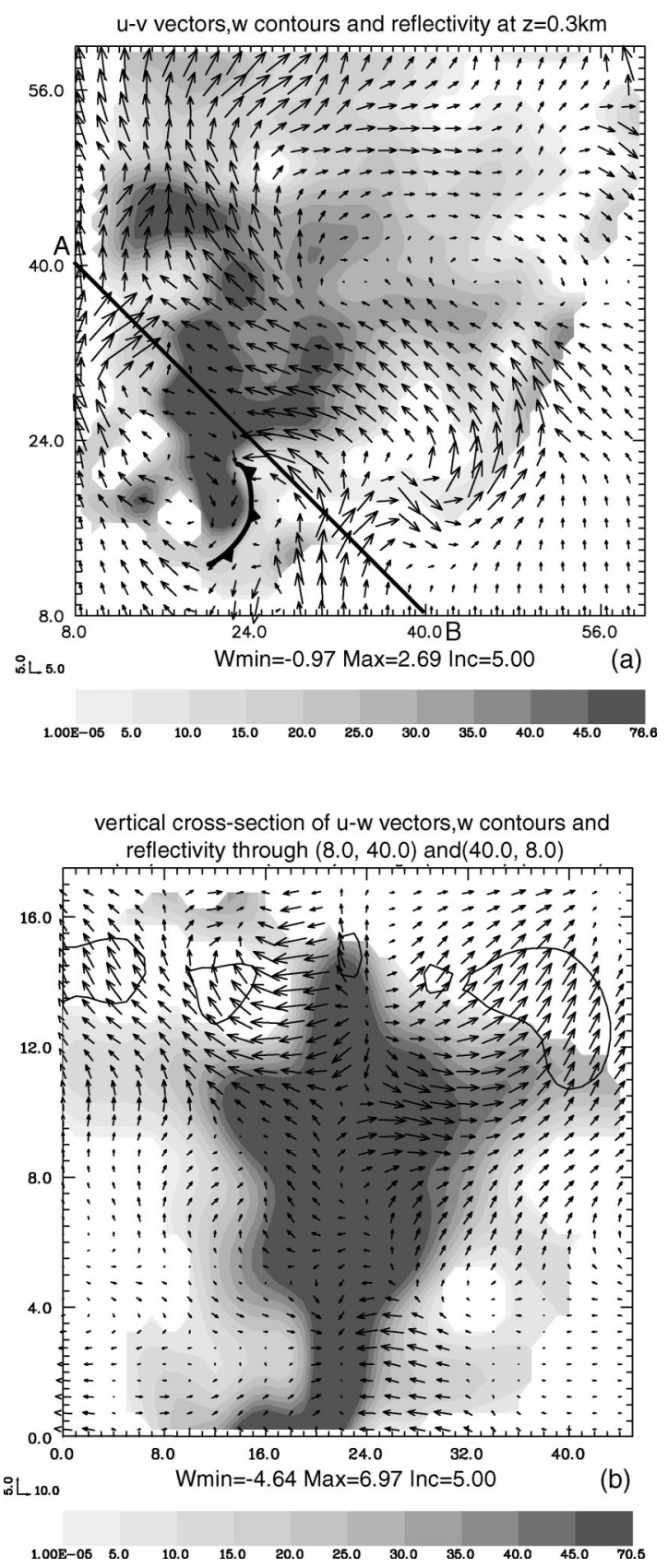

FIG. 10. As in Fig. 6, at 1643 CST, but for experiment without the anelastic mass conservation equation as weak constraint. (a) Horizontal cross section at $z=0.3 \mathrm{~km}$. (b) Vertical cross section through the A-B line in Fig. 6a.

step by adding a weak anelastic mass continuity constraint to the cost function. The use of a weak instead of strong constraint also leads to procedural simplicity in that the explicit solution of an elliptic equation, which would arise from the use of a strong constraint, is avoided. The latter tends to be sensitive to the specification of boundary conditions. This finding also agrees with the results of Xu and Qiu (1994) and Gao et al. (1999). 


\section{d. Experiment without recursive filter}

To test the important role of the background error covariance, we perform an additional experiment without employing the recursive filter. In this case, the influence of observational data is confined to the immediate surrounding grid points of observations. For our case of incomplete radar data coverage, the analysis is expected to be at least very noisy. Figure 11 shows the analyzed flow field and clearly illustrates that the result is quite unsatisfactory. At $5 \mathrm{~km}$ above the ground, the mesocyclone and mesoanticyclone are not well defined, though both still have some structure because the radar observations are dense at that level (not shown). The poor quality of the analysis is even more evident in a vertical slice (Fig. 11b), which reveals the presence of noise, especially in the anvil region and below $2 \mathrm{~km}$. Also, the analyzed vertical velocity extrema are onethird of those in the control experiment. We believe the poor analysis of horizontal velocity contributed to errors in horizontal divergence so that the mass continuity constraint also became ineffective.

This indicates that use of a recursive filter to model the background error covariance in the analysis plays a very important role in our variational method even though the filter used is simple, being isotropic and homogeneous. Work is under way to develop a more sophisticated inhomogeneous recursive filter to more realistically model the background error covariance, following the work of Purser et al. (2002a,b).

\section{Summary and concluding remarks}

In traditional dual-Doppler analysis, the need for explicitly integrating the mass continuity equation, as well as including "hole-filling" procedures, increases the solution sensitivity to boundary condition uncertainties. In addition, the separate interpolation from radar observation data points to analysis grid points in traditional methods can introduce errors. In this paper, we developed and tested a variational analysis scheme that is capable of retrieving and analyzing three-dimensional winds from dual-Doppler observations of convective storms. It was shown that the variational method has notable advantages over traditional dual-Doppler analysis techniques. The main conclusions can be summarized as follows.

1) The new method is able to incorporate radar data and background fields, along with mass continuity and other dynamic constraints, in a single cost function. By minimizing this cost function, an analysis with the desired fit to these constraints and observations is obtained in a single step.

2) The background error covariance matrix, though simple in this case, is modeled by a recursive filter, and the square root of the matrix is used as a preconditioning of minimization. Because the variational method preserves the radial nature of the ob-
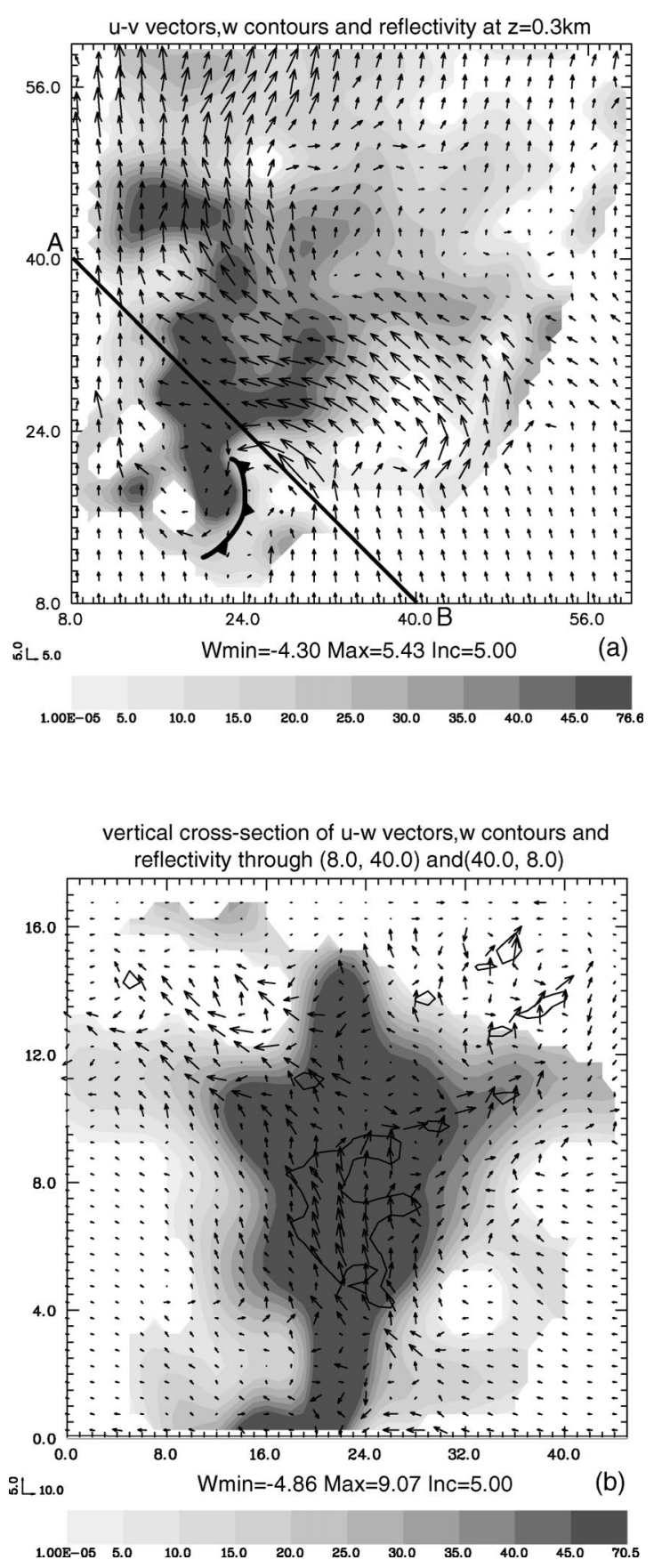

FIG. 11. As in Fig. 6, at 1643 CST, but for experiment without the recursive filters. (a) Horizontal cross section at $z=0.3 \mathrm{~km}$. (b) Vertical cross section through the A-B line in Fig. 6a.

servations, it allows for the forward interpolation from model grid to observation points so as to keep the observations close to their "raw" form.

3) Because no explicit integration of the anelastic mass continuity equation is involved, error accumulation during such integration is avoided. As a result, the method is less sensitive to boundary condition uncertainties. 
It is our plan to generalize our variational analysis procedure to include additional data sources, and to introduce additional dynamic constraints in the cost function so that thermodynamic fields are retrieved simultaneously with the winds. This procedure may also provide further improvements to the wind analysis.

Acknowledgments. This research was supported by NSF Grants ATM9909007 and ATM0129892, and by the FAA Grant NA17RJ1227-02, through NOAA. K. K. Droegemeier was also supported by NSF Grant ATM9981130. The first author appreciates the enlightening discussions with Dr. Qin Xu at the National Severe Storms Laboratory. Special thanks go to Dr. Stephen Weygandt for providing the Arcadia storm dataset. Graphics plots were generated using the ZXPLOT graphics package written by Ming Xue.

\section{REFERENCES}

Atkins, N. T., T. M. Weckwerth, and R. M. Wakimoto, 1995: Observations of the sea-breeze front during CaPE. Part II: Dual Doppler and aircraft analysis. Mon. Wea. Rev., 123, 944-969.

Atlas, D., R. C. Srivastava, and R. S. Sekhon, 1973: Doppler radar characteristics of precipitation at vertical incidence. Rev. Geophys. Space Phys., 11, 1-35.

Brandes, E. A., 1977: Flow in severe thunderstorms observed by dualDoppler radar. Mon. Wea. Rev., 105, 113-120.

_- 1981: Finestructure of the Del City-Edmond tornadic mesocirculation. Mon. Wea. Rev., 109, 635-647.

Browning, K. A., and R. Wexler, 1968: The determination of kinematic properties of a wind field using Doppler radar. J. Appl. Meteor., 7, 105-113.

Carbone, R. E., 1983: A severe frontal rainband. Part II: Tornado parent vortex circulation. J. Atmos. Sci., 40, 2639-2654.

Chong, M., J. Testud, and F. Roux, 1983a: Three-dimensional wind field analysis from dual-Doppler radar data. Part II: Minimizing the error due to temporal variation. J. Climate Appl. Meteor., 22, 1216-1226.

$\_,-$, and —_ 1983b: Three-dimensional wind field analysis from dual-Doppler radar data. Part III: Minimizing the error due to temporal variation. J. Climate Appl. Meteor., 22, 1227-1241.

Courtier, P., 1997: Dual formulation of four dimensional variational assimilation. Quart. J. Roy. Meteor. Soc., 123, 2449-2461.

— operational implementation of 4D-VAR, using an incremental approach. Quart. J. Roy. Meteor. Soc., 120, 1367-1387.

Doviak, R. J., P. S. Ray, R. G. Strauch, and L. J. Miller, 1976: Error estimation in wind fields derived from dual-Doppler radar measurement. J. Appl. Meteor., 15, 249-253.

Dowell, D. C., and H. B. Bluestein, 1997: The Arcadia, Oklahoma, storm of 17 May 1981: Analysis of a supercell during tornadogensis. Mon. Wea. Rev., 125, 2562-2582.

Droegemeier, K. K., 1997: The numerical prediction of thunderstorm: Challenges, potential benefits, and results from realtime operational tests. WMO Bull., 46, 324-336.

Ellis, S., 1997: Hole-filling data voids in meteorological fields. M.S. thesis, School of Meteorology, University of Oklahoma, $198 \mathrm{pp}$.

Foote, G. B., and P. S. duToit, 1969: Terminal velocity of raindrops aloft. J. Appl. Meteor., 8, 249-253.

Gal-Chen, T., 1982: Errors in fixed and moving frame of references: Applications for conventional and Doppler radar analysis. J. Atmos. Sci., 39, 2279-2300.

Gao, J., M. Xue, A. Shapiro, and K. K. Droegemeier, 1999: A variational analysis for the retrieval of three-dimensional mesoscale wind fields from two Doppler radars. Mon. Wea. Rev., 127, 2128-2142.

Gill, P. E., W. Murray, and M. H. Wright, 1981: Practical Optimization. Academic Press, 401 pp.

Hayden, C. M., and R. J. Purser, 1995: Recursive filter for objective analysis of meteorological fields: Applications to NESDIS operational processing. J. Appl. Meteor., 34, 3-15.

Houze, R. A., Jr., and P. V. Hobbs, 1982: Organization and structure of precipitating cloud systems. Advances in Geophysics, Vol. 24, Academic Press, 225-315.

Huang, X.-Y., 2000: Variational analysis using spatial filters. Mon. Wea. Rev., 128, 2588-2600.

Kessinger, C. J., P. S. Ray, and C. E. Hane, 1987: The Oklahoma squall line of 19 May 1977. Part I: A multiple Doppler analysis of convective and stratiform structure. J. Atmos. Sci., 44, 28402864.

Klemp, J. B., 1987: Dynamics of tornadic thunderstorms. Annu. Rev. Fluid Mech., 19, 1-33.

Lemon, L. R., and I. C. A. Doswell, 1979: Severe thunderstorm evolution and mesocyclone structure as related to tornadogenesis. Mon. Wea. Rev., 107, 1184-1197.

Lhermitte, R. M., and L. J. Miller, 1970: Doppler radar methodology for the observation of convective storms. Preprints, 14th Radar Meteorology Conf., Tucson, AZ, Amer. Meteor. Soc., 133-138.

Lin, Y., P. S. Ray, and K. W. Johnson, 1993: Initialization of a modeled convective storm using Doppler radar-derived fields. Mon. Wea. Rev., 121, 2757-2775.

Liou, Y.-C., 1999: Single radar recovery of cross-beam wind components using a modified moving frame of reference technique. J. Atmos. Oceanic Technol., 16, 1003-1016.

Lorenc, A. C., 1986: Analysis methods for numerical weather prediction. Quart. J. Roy. Meteor. Soc., 112, 1177-1194.

_ 1992: Iterative analysis using covariance functions and filters. Quart. J. Roy. Meteor. Soc., 118, 569-591.

Matejka, T., and D. Bartels, 1998: The accuracy of vertical air velocities from Doppler radar data. Mon. Wea. Rev., 126, 92-117.

Miller, L. J., and R. G. Strauch, 1974: A dual Doppler radar method for the determination of wind velocities within precipitating weather systems. Remote Sens. Environ., 3, 219-235.

_ storms: Specification of radar measurement errors. Preprints, 31st Conf. on Radar Meteorology, Seattle, WA, Amer. Meteor. Soc., 146-149.

Mohr, C. G., 1988: CEDRIC-Cartesian Space Data Processor. NCAR, Boulder, CO, 78 pp.

Navon, I. M., and D. M. Legler, 1987: Conjugate-gradient methods for large-scale minimization in meteorology. Mon. Wea. Rev., 115, 1479-1502.

Parsons, D. B., and R. A. Kropfli, 1990: Dynamics and fine structure of a microburst. J. Atmos. Sci., 47, 1674-1692.

Purser, R. J., and R. McQuigg, 1982: A successive correction analysis scheme using numerical filter. Met Office Tech. Note 154, 17 pp.

, W.-S. Wu, D. Parrish, and N. M. Roberts, 2003a: Numerical aspects of the application of recursive filters to variational statistical analysis. Part I: Spatially homogeneous and isotropic Gaussian covariances. Mon. Wea. Rev., 131, 1524-1535.

$\ldots, \ldots, \ldots$, and __ 2003b: Numerical aspects of the application of recursive filters to variational statistical analysis. Part II: Spatially inhomogeneous and anisotropic general covariances. Mon. Wea. Rev., 131, 1536-1548.

Ray, P. S., R. J. Doviak, G. B. Walker, D. Sirmans, J. Carter, and B. Bumgarner, 1975: Dual-Doppler observations of a tornadic storm. J. Appl. Meteor., 14, 1521-1530.

— C. C. Ziegler, W. Bumgarner, and R. J. Serafin, 1980: Singleand multiple-Doppler radar observations of tornadic storms. Mon. Wea. Rev., 108, 1607-1625.

, B. C. Johnson, K. W. Johnson, J. S. Bradberry, J. J. Stephens, K. K. Wagner, R. B. Wilhelmson, and J. B. Klemp, 1981: The 
morphology of several tornadic storms on 20 May 1977. J. Atmos. Sci., 38, 1643-1663.

Reinking, R. F., R. J. Doviak, and R. O. Gilmer, 1981: Clear-air roll vortices and turbulent motions as detected with an airborne gust probe and dual-Doppler radar. J. Appl. Meteor., 20, 678-685.

Shapiro, A., and J. J. Mewes, 1999: New formulations of dual-Doppler wind analysis. J. Atmos. Oceanic Technol., 16, 782-792.

Sun, J., and N. A. Crook, 1997: Dynamical and microphysical retrieval from Doppler radar observations using a cloud model and its adjoint. Part I: Model development and simulated data experiments. J. Atmos. Sci., 54, 1642-1661.

_, and _- 1998: Dynamical and microphysical retrieval from Doppler radar observations using a cloud model and its adjoint. Part II: Retrieval experiments of an observed Florida convective storm. J. Atmos. Sci., 55, 1642-1661.
Testud, J. D., and M. Chong, 1983: Three-dimensional wind field analysis from dual-Doppler radar data. Part I: Filtering, interpolation and differentiating the raw data. J. Climate Appl. Meteor., 22, 1204-1215.

Wu, W.-S., R. J. Purser, and D. Parrish, 2002: Three-dimensional variational analysis with spatially inhomogeneous covariances. Mon. Wea. Rev., 130, 2905-2916.

Xu, Q., and C. J. Qiu, 1994: Simple adjoint methods for singleDoppler wind analysis with a strong constraint of mass conservation. J. Atmos. Oceanic Technol., 11, 289-298.

— - and J. Gong, 2003: Background error covariance functions for Doppler radial-wind analysis. Quart. J. Roy. Meteor. Soc., 129, 1703-1720.

Ziegler, C., P. S. Ray, and N. C. Knight, 1983: Hail growth in an Oklahoma multi-cell storm. J. Atmos. Sci., 40, 1768-1791. 